Volume 25 (2019) 77-86

DOI: $10.24330 /$ ieja. 504118

\title{
ON THE EXTENDED TOTAL GRAPH OF MODULES OVER COMMUTATIVE RINGS
}

\author{
F. Esmaeili Khalil Saraei and E. Navidinia \\ Received: 23 February 2018; Revised: 7 September 2018; Accepted: 7 September 2018 \\ Communicated by A. Çiğdem Özcan
}

\begin{abstract}
Let $M$ be a module over a commutative $\operatorname{ring} R$ and $U$ a nonempty proper subset of $M$. In this paper, the extended total graph, denoted by $E T_{U}(M)$, is presented, where $U$ is a multiplicative-prime subset of $M$. It is the graph with all elements of $M$ as vertices, and for distinct $m, n \in M$, the vertices $m$ and $n$ are adjacent if and only if $r m+s n \in U$ for some $r, s \in R \backslash(U: M)$. We also study the two (induced) subgraphs $E T_{U}(U)$ and $E T_{U}(M \backslash U)$, with vertices $U$ and $M \backslash U$, respectively. Among other things, the diameter and the girth of $E T_{U}(M)$ are also studied.
\end{abstract}

Mathematics Subject Classification (2010): 13C13, 05C75, 13A15

Keywords: Total graph, prime submodule, multiplicative-prime subset

\section{Introduction}

Throughout this paper, $R$ is a commutative ring with nonzero identity and $M$ is a unitary $R$-module. Recently, there has been considerable attention in the literature to associating graphs with algebraic structures (see [1], [2], [3], [5], [6], [7], [8], and [9]). Anderson and Badawi in [4] defined a nonempty proper subset $H$ of $R$ to be a multiplicative-prime subset of $R$ if the following two conditions hold: (i) $r s \in H$ for every $r \in H$ and $s \in R$; (ii) if $r s \in H$ for some $r, s \in R$, then either $r \in H$ or

$s \in H$. They introduced the notion of the generalized total graph of a commutative ring $G T_{H}(R)$ with the vertices all elements of $R$, and two distinct vertices $x, y \in R$ are adjacent if and only if $x+y \in H$, where $H$ is a multiplicative-prime subset of $R$.

Let $R$ be a commutative ring and $U$ be a nonempty subset of an $R$-module $M$. The subset $\{r \in R: r M \subseteq U\}$ will be denoted by $\left(U:_{R} M\right)$ or $(U: M)$. It is clear that if $U$ is a submodule of $M$, then $(U: M)$ is an ideal of $R$. We define a nonempty subset $U$ of $M$ to be a multiplicative-prime subset of $M$ if the following two conditions hold: (i) $r m \in U$ for every $r \in R$ and $m \in U$; (ii) if $s x \in U$ for some $s \in R$ and $x \in M$, then $x \in U$ or $s \in(U: M)$. Note that if $U$ is a 
multiplicative-prime submodule of $M$, then $U$ is necessarily a prime submodule of $M$. One can show that if $U$ is a multiplicative-prime subset of $M$, then $(U: M)$ is a multiplicative-prime subset of $R$.

The total graph of a module $M$ with respect to a multiplicative-prime subset $U$ (denoted by $G T_{U}(M)$ ) was introduced in [10]. The set of vertices of $G T_{U}(M)$ is all elements of $M$, and two distinct vertices $m$ and $n$ adjacent whenever $m+n \in U$. In this paper, we introduce an extension of the graph $G T_{U}(M)$, denoted by $E T_{U}(M)$, such that its vertex set consists of all elements of $M$ and for distinct $m, n \in M$, the vertices $m$ and $n$ are adjacent if and only if $r m+s n \in U$ for some $r, s \in R \backslash(U: M)$, where $U$ is a multiplicative-prime subset of $M$.

Let $E T_{U}(U)$ be the (induced) subgraph of $E T_{U}(M)$ with vertex set $U$, and let $E T_{U}(M \backslash U)$ be the (induced) subgraph $E T_{U}(M)$ with vertices consisting of $M \backslash U$. Obviously, the total graph $G T_{U}(M)$ is a subgraph of $E T_{U}(M)$. It follows that each edge (path) of $G T_{U}(M)$ is an edge (path) of $E T_{U}(M)$. The study of $E T_{U}(M)$ breaks naturally into two cases depending on whether or not $U$ is a submodule of $M$. In the second section, we handle the case when $U$ is a submodule of $M$; in the third section, we do the case when $U$ is not a submodule of $M$. For every case, we characterize the girth and diameter of $E T_{U}(M), E T_{U}(U)$ and $E T_{U}(M \backslash U)$.

We begin with some notation, and definitions. For a graph $\Gamma$, by $E(\Gamma)$ and $V(\Gamma)$, we mean the set of all edges and vertices, respectively. We recall that a graph is connected if there exists a path connecting any two of its distinct vertices. At the other extreme, we say that a graph is totally disconnected if no two vertices of this graph are adjacent. The distance between two distinct vertices $a$ and $b$, denoted by $\mathrm{d}(a, b)$, is the length of a shortest path connecting them (if such a path does not exist, then $d(a, b)=\infty)$. We also define $d(a, a)=0$. The diameter of a graph $\Gamma$, denoted by $\operatorname{diam}(\Gamma)$, is equal to $\sup \{d(a, b): a, b \in V(\Gamma)\}$. A graph is complete if it is connected with diameter less than or equal to one. The girth of a graph $\Gamma$, denoted by $\operatorname{gr}(\Gamma)$, is the length of a shortest cycle in $\Gamma$, provided $\Gamma$ contains a cycle; otherwise; $\operatorname{gr}(\Gamma)=\infty$. We denote the complete graph on $n$ vertices by $K^{n}$ and the complete bipartite graph on $m$ and $n$ vertices by $K^{m, n}$ (we allow $m$ and $n$ to be infinite cardinals). For a graph $\Gamma$, the degree of a vertex $v$ in $\Gamma$, denoted by $\operatorname{deg}(v)$, is the number of edges of $\Gamma$ incident with $v$. We say that two (induced) subgraphs $\Gamma_{1}$ and $\Gamma_{2}$ of $\Gamma$ are disjoint if $\Gamma_{1}$ and $\Gamma_{2}$ have no common vertices and no vertex of $\Gamma_{1}$ is adjacent(in $\left.\Gamma\right)$ to some vertex of $\Gamma_{2}$. 


\section{The case when $U$ is a submodule of $M$}

In this section, we study the case when $U$ is a submodule of $M$. It is clear that if $U$ is a submodule of $M$, then $U$ is a prime submodule of $M$. If $U=M$, then it is clear that $E T_{U}(M)$ is a complete graph and $E T_{U}(M)$ is a disconnected graph when $U=0$ and $|M| \geq 2$. So we may assume that $U \neq 0$ and $U \neq M$.

First, we begin with the following example that shows we may have $\operatorname{ET}_{U}(M) \neq$ $G T_{U}(M)$.

Example 2.1. Let $R=\mathbb{Z}$ and $M=\mathbb{Z}_{10}$. Set $U=\{\overline{0}, \overline{5}\}$. It is clear that $U$ is a submodule of $M$ and $(U: M)=5 \mathbb{Z}$. Since $\overline{1}+\overline{3}=\overline{4} \notin U$, so $\overline{1}-\overline{3}$ is not an edge in $G T_{U}(M)$. But $2(\overline{1})+1(\overline{3})=\overline{5} \in U$ and $2,1 \in R \backslash(U: M)$. Thus $\overline{1}-\overline{3}$ is an edge in $E T_{U}(M)$. Hence $E T_{U}(M) \neq G T_{U}(M)$.

The main goal of this section is a general structure theorem (Theorem 2.4) for $E T_{U}(M \backslash U)$ when $U$ is a submodule of $M$. But first, we record the trivial observation that if $U$ is a submodule of $M$, then $E T_{U}(U)$ is a complete subgraph of $E T_{U}(M)$ and is disjoint from $E T_{U}(M \backslash U)$. Thus we will concentrate on the subgraph $E T_{U}(M \backslash U)$ throughout this section.

Theorem 2.2. Let $M$ be a module over a commutative ring $R$ and $U$ be a prime submodule of $M$. Then $E T_{U}(U)$ is a complete subgraph of $E T_{U}(M)$ and is disjoint from $E T_{U}(M \backslash U)$. In particular, $E T_{U}(U)$ is connected and $E T_{U}(M)$ is disconnected.

Proof. Let $m, n \in U$. Then it is clear that $m+n \in U$ since $U$ is a submodule of $M$. If $x \in U$ is adjacent to $y \in M \backslash U$, then $r x+s y \in U$ for some $r, s \in R \backslash(U: M)$. This implies that $s y \in U$; so $y \in U$ or $s \in(U: M)$ since $U$ is a prime submodule, which is a contradiction. The "in particular" statement is clear.

Theorem 2.3. Let $M$ be a module over a commutative ring $R$ and $U$ be a prime submodule of $M$. Let $G$ be an induced subgraph of $E T_{U}(M \backslash U)$, and $m$ and $m^{\prime}$ be distinct nonadjacent vertices of $G$ that are connected by a path in $G$. Then there exists a path in $G$ of length 2 between $m$ and $m^{\prime}$. In particular, if $E T_{U}(M \backslash U)$ is connected, then $\operatorname{diam}\left(E T_{U}(M \backslash U)\right) \leq 2$.

Proof. (1) Let $m_{1}, m_{2}, m_{3}$ and $m_{4}$ be distinct vertices of $G$. It suffices to show that if there is a path $m_{1}-m_{2}-m_{3}-m_{4}$ from $m_{1}$ to $m_{4}$, then $m_{1}$ and $m_{4}$ are adjacent. Now, $r_{1} m_{1}+r_{2} m_{2}, r_{2}^{\prime} m_{2}+r_{3}^{\prime} m_{3}, r_{3} m_{3}+r_{4} m_{4} \in U$ for some $r_{1}, r_{2}, r_{2}^{\prime}, r_{3}^{\prime}, r_{3}, r_{4} \in$ $R \backslash(U: M)$. Hence $\left(r_{1} r_{3} r_{2}^{\prime}\right) m_{1}+\left(r_{2} r_{3}^{\prime} r_{4}\right) m_{4}=r_{3} r_{2}^{\prime}\left(r_{1} m_{1}+r_{2} m_{2}\right)-r_{2} r_{3}\left(r_{2}^{\prime} m_{2}+\right.$ 
$\left.r_{3}^{\prime} m_{3}\right)+r_{2} r_{3}^{\prime}\left(r_{3} m_{3}+r_{4} m_{4}\right) \in U$, and $r_{1} r_{3} r_{2}^{\prime}, r_{2} r_{3}^{\prime} r_{4} \notin(U: M)$ since $(U: M)$ is a prime ideal of $R$. Thus $m_{1}$ and $m_{4}$ are adjacent. So if $E T_{U}(M \backslash U)$ is connected, then $\operatorname{diam}\left(E T_{U}(M \backslash U)\right) \leq 2$.

Now, we give the main theorem of this section. Since $E T_{U}(U)$ is a complete subgraph of $E T_{U}(M)$ by Theorem 2.2, the next theorem gives a complete description of $E T_{U}(M \backslash U)$. Let $|U|=\alpha$. We allow $\alpha$ to be an infinite cardinal. Compare the next theorem with [10, Theorem 3.5].

Theorem 2.4. Let $M$ be a module over a commutative ring $R, U$ be a prime submodule of $M$, and $|U|=\alpha$.

(1) If $r+s \in(U: M)$ for some $r, s \in R \backslash(U: M)$, then $E T_{U}(M \backslash U)$ is the union of complete subgraphs.

(2) If $r+s \notin(U: M)$ for all $r, s \in R \backslash(U: M)$, then $E T_{U}(M \backslash U)$ is the union of totally disconnected subgraphs and some connected subgraphs.

Proof. (1) Suppose that $r+s \in(U: M)$ for some $r, s \in R \backslash(U: M)$. For $m, m^{\prime} \in M \backslash U$, we write $m \sim m^{\prime}$ if and only if $t m+t^{\prime} m^{\prime} \in U$ and $t+t^{\prime} \in(U: M)$ for some $t, t^{\prime} \in R \backslash(U: M)$. It is straightforward to check that $\sim$ is an equivalence relation on $M \backslash U$ since $U$ is a prime submodule. For $m \in M \backslash U$, we denote the equivalence class which contains $m$ by $[m]$. Now let $m \in M \backslash U$. If $[m]=\{m\}$, then $r\left(m+u_{1}\right)+s\left(m+u_{2}\right)=(r+s) m+r u_{1}+s u_{2} \in U$ for every $u_{1}, u_{2} \in U$ since $r+s \in(U: M)$. Then $m+U$ is a complete subgraph of $E T_{U}(M \backslash U)$ with at most $\alpha$ vertices. Now let $|[m]|=\nu$ and $m^{\prime} \in[m]$. Then $t m+t^{\prime} m^{\prime} \in U$ and $t+t^{\prime} \in(U: M)$ for some $t, t^{\prime} \in R \backslash(U: M)$. So $t\left(m+u_{1}\right)+t^{\prime}\left(m^{\prime}+u_{2}\right)=t m+t^{\prime} m^{\prime}+t u_{1}+t^{\prime} u_{2} \in U$ for every $u_{1}, u_{2} \in U$. Thus $m+U$ is part of the complete graph $k^{\mu}$, where $\mu \leq \alpha \nu$. (2) Assume that $r+s \notin(U: M)$ for all $r, s \in R \backslash(U: M)$. Let

$$
A_{m}=\left\{m^{\prime} \in M \backslash U: r m+s m^{\prime} \in U \text { for some } r, s \in R \backslash(U: M)\right\}
$$

be the set of all vertices adjacent to $m$. If $A_{m}=\emptyset$, then $p m+q m^{\prime} \notin U$ for every $m^{\prime} \in M \backslash U$ and every $p, q \in R \backslash(U: M)$. In this case, we show that $m+U$ is a totally disconnected subgraph of $E T_{U}(M \backslash U)$. If $r\left(m+m_{1}\right)+s\left(m+m_{2}\right) \in U$ for some $r, s \in R \backslash(U: M)$ and $m_{1}, m_{2} \in U$, then $(r+s) m \in U$. Since $U$ is a prime submodule of $M$ and $m \notin U$, then $r+s \in(U: M)$, which is a contradiction. Therefore $m+U$ is a totally disconnected subgraph of $E T_{U}(M \backslash U)$. Now, we may assume that $A_{m} \neq \emptyset$. Then $r m+s m^{\prime} \in U$ for some $r, s \in R \backslash(U: M)$ and $m^{\prime} \in M \backslash U$. Thus $r\left(m+u_{1}\right)+s\left(m^{\prime}+u_{2}\right)=r m+s m^{\prime}+r u_{1}+s u_{2} \in U$ for every $u_{1}, u_{2} \in U$; hence each element of $m+U$ is adjacent to each element of $m^{\prime}+U$. 
ON THE EXTENDED TOTAL GRAPH OF MODULES OVER COMMUTATIVE RINGS 81

If $\left|A_{m}\right|=\nu$, then we have a connected subgraph of $E T_{U}(M \backslash U)$ with at most $\alpha \nu$ vertices. So $E T_{U}(M \backslash U)$ is the union of totally disconnected subgraphs and some connected subgraphs.

Now it is easy to compute the diameter and the girth of $E T_{U}(M \backslash U)$ using Theorem 2.4.

Theorem 2.5. Let $M$ be a module over a commutative ring $R$ such that $U$ is a prime submodule of $M$.

(1) $\operatorname{diam}\left(E T_{U}(M \backslash U)\right)=0$ if and only if $U=\{0\}$ and $|M|=2$.

(2) $\operatorname{diam}\left(E T_{U}(M \backslash U)\right)=1$ if and only if either $|M \backslash U|=1$ and $r+s \notin(U: M)$ for some $r, s \in R \backslash(U: M)$ or $|M \backslash U|=2, r+s \in(U: M)$ for every $r, s \in R \backslash(U: M)$ and $x+y \in U$ for some distinct elements $x, y \in M \backslash U$.

(3) $\operatorname{diam}\left(E T_{U}(M \backslash U)\right)=2$ if and only if $|M \backslash U|=2, r+s \notin(U: M)$ for every $r, s \in R \backslash(U: M), x+y \in U$ for some distinct elements $x, y \in M \backslash U$ and $|m+U| \geq 2$ for some $m \in M \backslash U$.

(4) Otherwise, $\operatorname{diam}\left(E T_{U}(M \backslash U)\right)=\infty$.

Proof. (1) If $\operatorname{diam}\left(E T_{U}(M \backslash U)\right)=0$, then $E T_{U}(M \backslash U)$ is a complete graph $K^{1}$, and so $|U|=|M / U|=1$ by Theorem 2.4. Hence $U=\{0\}$ and $|M|=2$. Now, let $U=\{0\}$ and $M=\{0, m\}$. Then $m+U$ is a single graph $K^{1}$. So $\operatorname{diam}\left(E T_{U}(M \backslash U)\right)=0$.

(2) It is clear that $E T_{U}(M \backslash U)$ is a complete graph if and only if $\operatorname{diam}\left(E T_{U}(M \backslash\right.$ $U)=1$. So the proof is clear by Theorem 2.4.

(3) If $\operatorname{diam}\left(E T_{U}(M \backslash U)\right)=2$, then $E T_{U}(M \backslash U)$ is a complete bipartite graph $K^{m, n}$ such that $m \geq 2$ or $n \geq 2$. Thus $r+s \notin(U: M)$ for every $r, s \in R \backslash(U: M)$ by Theorem 2.4. Therefore $|M \backslash U|=2$ and $x+y \in U$ for some $x, y \in M \backslash U$. Since $m \geq 2$ or $n \geq 2$, we have $|x+U| \geq 2$ or $|y+U| \geq 2$. Conversely, let $r+s \notin(U: M)$ for every $r, s \in R \backslash(U: M)$ and $|M \backslash U|=2$. Then $M=$ $U \cup(x+U) \cup(y+U)$ and $E T_{U}(M \backslash U)$ is a complete bipartite graph since $x+y \in U$. Hence $\operatorname{diam}\left(E T_{U}(M \backslash U)\right)=2$, since $|x+U| \geq 2$ or $|y+U| \geq 2$.

Theorem 2.6. Let $M$ be a module over a commutative ring $R$ such that $U$ is a prime submodule of $M$. Then $\operatorname{gr}\left(E T_{U}(M \backslash U)\right)=3,4$, or $\infty$. In particular, $\operatorname{gr}\left(E T_{U}(M \backslash U)\right) \leq 4$ if $E T_{U}(M \backslash U)$ contains a cycle.

Proof. Assume that $E T_{U}(M \backslash U)$ contains a cycle. Then $E T_{U}(M \backslash U)$ is not a totally disconnected graph; so by the proof of Theorem 2.4, $E T_{U}(M \backslash U)$ has either a complete or a complete bipartite subgraph. Therefore it must contain either a 3-cycle or 4-cycle. Thus $\operatorname{gr}\left(E T_{U}(M \backslash U)\right) \leq 4$. 
Theorem 2.7. Let $M$ be a module over a commutative ring $R$ such that $U$ is a prime submodule of $M$.

(1) $\operatorname{gr}\left(E T_{U}(M \backslash U)\right)=3$ if and only if $r+s \in(U: M)$ and $|y+U| \geq 3$ for some $r, s \in R \backslash(U: M)$ and $y \in M \backslash U$.

(2) $\operatorname{gr}\left(E T_{U}(M \backslash U)\right)=4$ if and only if $r+s \notin(U: M)$ for every $r, s \in R \backslash(U:$ $M)$ and $p m+q m^{\prime} \in U$ for some $m, m^{\prime} \in M \backslash U$ and $p, q \in R \backslash(U: M)$.

(3) Otherwise, $\operatorname{gr}\left(E T_{U}(M \backslash U)\right)=\infty$.

Proof. (1) Assume that $\operatorname{gr}\left(E T_{U}(M \backslash U)\right)=3$. Then by Theorem 2.4, $E T_{U}(M \backslash U)$ is a complete graph $K^{\lambda}$, where $\lambda \geq 3$. Then $r+s \in(U: M)$ for some $r, s \in R \backslash(U$ : $M)$ and $|y+U| \geq 3$ for some $y \in M \backslash U$ by Theorem 2.4.

(2) If $\operatorname{gr}\left(E T_{U}(M)\right)=4$, then $E T_{U}(M \backslash U)$ has a complete bipartite subgraph. So $r+s \notin(U: M)$ for every $r, s \in R \backslash(U: M)$ and $p m+q m^{\prime} \in U$ for some $m, m^{\prime} \in M \backslash U$ and $p, q \in R \backslash(U: M)$ by Theorem 2.4.

The other implications of (1) and (2) follows directly from Theorem 2.4.

We end this section with the following theorem.

Theorem 2.8. Let $M$ be a module over a commutative ring $R$ such that $U$ is a prime submodule of $M$.

(1) $\operatorname{gr}\left(E T_{U}(M)\right)=3$ if and only if $|U| \geq 3$.

(2) $\operatorname{gr}\left(E T_{U}(M)\right)=4$ if and only if $r+s \notin(U: M)$ for every $r, s \in R \backslash(U: M)$, $|U|<3$, and $p m+q m^{\prime} \in U$ for some $m, m^{\prime} \in M \backslash U$ and $p, q \in R \backslash(U: M)$.

(3) Otherwise, $\operatorname{gr}\left(E T_{U}(M)\right)=\infty$.

Proof. (1) This follows from Theorem 2.2.

(2) Assume that $\operatorname{gr}\left(E T_{U}(M)\right)=4$. Since $\operatorname{gr}\left(E T_{U}(U)\right)=3$ or $\infty$, then $\operatorname{gr}\left(E T_{U}(M \backslash\right.$ $U))=4$. Therefore $r+s \notin(U: M)$ for every $r, s \in R \backslash(U: M)$ and $p m+q m^{\prime} \in U$ for some $m, m^{\prime} \in M \backslash U$ and $p, q \in R \backslash(U: M)$ by Theorem 2.7. On the other hand, $\operatorname{gr}\left(E T_{U}(M)\right) \neq 3$; so $|U|<3$. The other implication follows from Theorem 2.4 .

\section{The case when $U$ is not a submodule of $M$}

In this section, we study $E T_{U}(M)$ when the multiplicative-prime subset $U$ is not a submodule of $M$. Since $U$ is always closed under multiplication by elements of $R$, this just means that $0 \in U$ and there are distinct $x, y \in U$ such that $x+y \in M \backslash U$.

First, we begin with the following example that shows we may have $\operatorname{ET}_{U}(M) \neq$ $G T_{U}(M)$. 
Example 3.1. Let $R=M=\mathbb{Z}$. Set $U=4 \mathbb{Z} \cup 6 \mathbb{Z}$. It is clear that $(U: M)=U$ and $U$ is not a submodule of $M$ since $4,6 \in U$, but $4+6=10 \notin U$. So $4-6$ is not an edge in $G T_{U}(M)$. But $2(4)+2(6)=20 \in U$ and $2 \in R \backslash U$. Thus $4-6$ is an edge in $E T_{U}(M)$. Hence $E T_{U}(M) \neq G T_{U}(M)$.

Now, we have the following theorem that shows $E T_{U}(U)$ is always connected (but never complete), $E T_{U}(U)$ and $E T_{U}(M \backslash U)$ are never disjoint subgraphs of $E T_{U}(M)$, and $E T_{U}(M)$ is connected when $E T_{U}(M \backslash U)$ is connected.

Theorem 3.2. Let $M$ be a module over a commutative ring $R$ such that $U$ is a multiplicative-prime subset of $M$ that is not a submodule of $M$. Then the following hold:

(1) $E T_{U}(U)$ is connected with $\operatorname{diam}\left(E T_{U}(U)\right)=2$.

(2) Some vertex of $E T_{U}(U)$ is adjacent to a vertex of $E T_{U}(M \backslash U)$. In particular, the subgraphs $E T_{U}(U)$ and $E T_{U}(M \backslash U)$ are not disjoint.

(3) If $E T_{U}(M \backslash U)$ is connected, then $E T_{U}(M)$ is connected.

Proof. (1) Let $u \in U^{*}=U \backslash\{0\}$. Then $u$ is adjacent to 0 . Thus $u-0-u^{\prime}$ is a path in $E T_{U}(U)$ of length two between any two distinct $u, u^{\prime} \in U^{*}$. Moreover, there exist nonadjacent $u, u^{\prime} \in U^{*}$ since $U$ is not a submodule of $M$; thus $\operatorname{diam}\left(E T_{U}(U)\right)=2$. (2) Since $U$ is not a submodule of $M$, there exist distinct $m, n \in U^{*}$ such that $m+n \notin U$. Then $-m \in U$ and $m+n \notin U$ are adjacent vertices in $E T_{U}(M)$. Finally, the "in particular" statement is clear.

(3) $E T_{U}(U)$ and $E T_{U}(M \backslash U)$ are connected, and there is an edge between $E T_{U}(U)$ and $E T_{U}(M \backslash U)$; thus $E T_{U}(M)$ is connected.

We determine when $E T_{U}(M)$ is connected and compute $\operatorname{diam}\left(E T_{U}(M)\right)$ with the following theorem. Compare the next theorem with [10, Theorem 4.2].

Theorem 3.3. Let $M$ be a module over a commutative ring $R$ such that $U$ is a multiplicative-prime subset of $M$ that is not a submodule of $M$. Then $E T_{U}(M)$ is connected if and only if for every $m \in M$ there exists $r \in R \backslash(U: M)$ such that $r m \in\langle U\rangle$.

Proof. Suppose that $E T_{U}(M)$ is connected and $m \in M$. Then there exists a path $0-x_{1}-x_{2}-\cdots-x_{n}-m$ from 0 to $m$ in $E T_{U}(M)$. Thus

$$
r_{1} x_{1}, r_{2} x_{1}+r_{3} x_{2}, \ldots, r_{2 n-2} x_{n-1}+r_{2 n-1} x_{n}, r_{2 n} x_{n}+s m \in U
$$

for some $r_{1}, r_{2}, \ldots, r_{2 n}, s \in R \backslash(U: M)$. Then

$$
s r_{1} r_{3} r_{5} \cdots r_{2 n-1} m=\left(r_{1} r_{3} r_{5} \cdots r_{2 n-1}\right)\left(s m+r_{2 n} x_{n}\right)-
$$




$$
\begin{gathered}
\left(r_{1} r_{3} r_{5} \cdots r_{2 n-3} r_{2 n}\right)\left(r_{2 n-2} x_{n-1}+r_{2 n-1} x_{n}\right)+\cdots \\
-\left(r_{1} r_{3} \cdots r_{2 n-2 k-5} r_{2 n-2 k-3} r_{2 n-2 k} r_{2 n-2 k-2} \cdots r_{2 n}\right)\left(r_{2 n-2 k-1} x_{n-k}+r_{2 n-2 k-2} x_{n-(k+1)}\right) \\
+\left(r_{1} r_{3} \cdots r_{2 n-2 k-5} r_{2 n-2 k-2} r_{2 n-2 k} r_{2 n-2 k-2} \cdots r_{2 n}\right)\left(r_{2 n-2 k-3} x_{n-(k+1)}+r_{2 n-2 k-4} x_{n-(k+2)}\right) \\
\cdots-\left(r_{2} r_{4} r_{6} \cdots r_{2 n}\right)\left(r_{1} x_{1}\right) \in\langle U\rangle
\end{gathered}
$$

Since $(U: M)$ is a multiplicative-prime subset of $R$, we have $r=s r_{1} r_{3} r_{5} \cdots r_{2 n-1} \in$ $R \backslash(U: M)$ and $r m \in\langle U\rangle$. Conversely, suppose that for every $m \in M$ there exists $r \in R \backslash(U: M)$ such that $r m \in\langle U\rangle$. We show that for each $0 \neq m \in M$, there exists a path in $E T_{U}(M)$ from 0 to $m$. By assumption, there are elements $u_{1}, u_{2}, \ldots, u_{n} \in$ $U$ such that $r m=u_{1}+u_{2}+\cdots+u_{n}$. Set $y_{0}=0$ and $y_{k}=(-1)^{n+k}\left(u_{1}+u_{2}+\cdots+u_{k}\right)$ for each integer $k$ with $1 \leq k \leq n$. Then $y_{k}+y_{k+1}=(-1)^{n+k+1} u_{k+1} \in U$ for each integer $1 \leq k \leq n-1$. Also, $y_{n-1}+r m=y_{n-1}+y_{n}=u_{n} \in U$. Thus $0-y_{1}-y_{2}-\cdots-y_{n-1}-m$ is a path from 0 to $m$ in $E T_{U}(M)$. Now, let $0 \neq x, y \in M$. Then by the preceding argument, there are paths from $x$ to 0 and 0 to $y$ in $E T_{U}(M)$. Hence there is a path from $x$ to $y$ in $E T_{U}(M)$. So $E T_{U}(M)$ is connected.

Theorem 3.4. Let $M$ be a module over a commutative ring $R$ such that $U$ is a multiplicative-prime subset of $M$ that is not a submodule of $M$ and for every $m \in M$ there exists $r \in R \backslash(U: M)$ such that $r m \in\langle U\rangle$. Let $n \geq 2$ be the least integer such that $\langle U\rangle=<m_{1}, m_{2}, \ldots, m_{n}>$ for some $m_{1}, m_{2}, \ldots, m_{n} \in U$. Then $\operatorname{diam}\left(E T_{U}(M)\right) \leq n$.

Proof. Let $m$ and $m^{\prime}$ be distinct elements in $M$. We show that there exists a path from $m$ to $m^{\prime}$ in $E T_{U}(M)$ with length at most $n$. By hypothesis, $r m, r^{\prime} m^{\prime} \in\langle U\rangle$ for some $r, r^{\prime} \in R \backslash(U: M)$; so we can write $r m=\sum_{i=1}^{n} r_{i} m_{i}$ and $r^{\prime} m^{\prime}=\sum_{i=1}^{n} s_{i} m_{i}$ for some $r_{i}, s_{i} \in R$. Define $x_{0}=m$ and $x_{k}=(-1)^{k}\left(\sum_{i=k+1}^{n} r_{i} m_{i}+\sum_{i=1}^{k} s_{i} m_{i}\right)$; so $x_{k}+x_{k+1}=(-1)^{k}\left(r_{k+1}-s_{k+1}\right) m_{k+1} \in U$ for each integer $k$ with $1 \leq k \leq n-1$. On the other hand, $r m+x_{1}=\left(r_{1}-s_{1}\right) m_{1} \in U$ and $r^{\prime} m^{\prime}+(-1)^{n} x_{n-1}=\left(s_{n}-r_{n}\right) m_{n} \in$ $U$. So $m-x_{1}-x_{2}-\cdots-x_{n-1}-m^{\prime}$ is a path from $m$ to $m^{\prime}$ in $E T_{U}(M)$ with length at most $n$ since $1,(-1)^{n} \notin(U: M)$.

We end the paper with the following theorem.

Theorem 3.5. Let $M$ be a module over a commutative ring $R$ such that $U$ is a multiplicative-prime subset of $M$ that is not a submodule of $M$. Then the following hold:

(1) Either $\operatorname{gr}\left(E T_{U}(U)\right)=3$ or $\operatorname{gr}\left(E T_{U}(U)\right)=\infty$.

(2) If $\operatorname{gr}\left(E T_{U}(M)\right)=4$, then $\operatorname{gr}\left(E T_{U}(U)\right)=\infty$. 
Proof. (1) If $r m+s m^{\prime} \in U$ for some distinct $m, m^{\prime} \in U \backslash\{0\}$ and $r, s \in R \backslash(U: M)$, then $0-m-m^{\prime}-0$ is a cycle of length 3 in $E T_{U}(U)$; so $\operatorname{gr}\left(E T_{U}(U)\right)=3$. Otherwise, $r m+s m^{\prime} \in M \backslash U$ for all distinct $m, m^{\prime} \in U \backslash\{0\}$ and all elements $r, s \in R \backslash(U: M)$. Therefore in this case, each nonzero element $m \in U$ is adjacent to 0 , and no two distinct $m, m^{\prime} \in U \backslash\{0\}$ are adjacent. Thus $\operatorname{gr}\left(E T_{U}(U)\right)=\infty$.

(2) If $\operatorname{gr}\left(E T_{U}(M)\right)=4$, then it is clear $\operatorname{gr}\left(E T_{U}(U)\right) \neq 3$. So $\operatorname{gr}\left(E T_{U}(U)\right)=\infty$ by part (1) above.

Acknowledgement. The authors are deeply grateful to the referee for a careful reading of this manuscript and his valuable suggestions.

\section{References}

[1] D. D. Anderson and M. Naseer, Beck's coloring of a commutative ring, J. Algebra, 159(2) (1993), 500-514.

[2] D. F. Anderson, M. C. Axtell and J. A. Stickles, Jr., Zero-divisor graphs in commutative rings, Commutative Algebra, Noetherian and Non-Noetherian Perspectives, eds. M. Fontana, S. E. Kabbaj, B. Olberding and I. Swanson, Springer-Verlag, New York, (2011), 23-45.

[3] D. F. Anderson and A. Badawi, The total graph of a commutative ring, J. Algebra, 320(7) (2008), 2706-2719.

[4] D. F. Anderson and A. Badawi, The generalized total graph of a commutative ring, J. Algebra Appl., 12(5) (2013), 1250212 (18 pp).

[5] D. F. Anderson and P. S. Livingston, The zero-divisor graph of a commutative ring, J. Algebra, 217(2) (1999), 434-447.

[6] D. F. Anderson and S. B. Mulay, On the diameter and girth of a zero-divisor graph, J. Pure Appl. Algebra, 210(2) (2007), 543-550.

[7] I. Beck, Coloring of commutative rings, J. Algebra, 116(1) (1988), 208-226.

[8] S. Ebrahimi Atani and S. Habibi, The total torsion element graph of a module over a commutative ring, An. Stiint. Univ. "Ovidius" Constanta Ser. Mat., 19(1) (2011), 23-34.

[9] F. Esmaeili Khalil Saraei, The total torsion element graph without the zero element of modules over commutative rings, J. Korean Math. Soc., 51(4) (2014), 721-734.

[10] F. Esmaeili Khalil Saraei, H. Heydarinejad Astaneh and R. Navidinia, The total graph of a module with respect to multiplicative-prime subsets, Rom. J. Math. Comput. Sci., 4(2) (2014), 151-166. 
F. Esmaeili Khalil Saraei (Corresponding Author)

Fouman Faculty of Engineering

College of Engineering

University of Tehran

P.O. Box 43515-1155, Fouman

43516-66456, Guilan, Iran

e-mail: f.esmaeili.kh@ut.ac.ir

\section{E. Navidinia}

Department of Mathematics

Faculty of Mathematical Sciences

University of Guilan

P.O.Box 1914, Rasht, Iran

e-mail: elnaz.navidinia@yahoo.com 